\title{
Channel-Lining Residues of the AMPA Receptor M2 Segment: Structural Environment of the Q/R Site and Identification of the Selectivity Filter
}

\author{
Thomas Kuner, ${ }^{1,2}$ Christine Beck, ${ }^{1}$ Bert Sakmann, ${ }^{2}$ and Peter H. Seeburg ${ }^{1}$ \\ ${ }^{1}$ Abteilung Molekulare Neurobiologie, ${ }^{2}$ Abteilung Zellphysiologie, Max-Planck Institut für Medizinische Forschung, 69120 \\ Heidelberg, Germany
}

In AMPA receptor channels, a single amino acid residue (Q/R site) of the M2 segment controls permeation of calcium ions, single-channel conductance, blockade by intracellular polyamines, and permeation of anions. The structural environment of the $Q / R$ site and its positioning with regard to a narrow constriction were probed with the accessibility of substituted cysteines to positively and negatively charged methanethiosulfonate reagents, applied from the extracellular and cytoplasmic sides of the channel. The accessibility patterns confirm that the M2 segment forms a pore loop with the Q/R site positioned at the tip of the loop (position 0 ) facing the extracellular vestibule. Cytoplasmically accessible residues on the $\mathrm{N}$ - and $\mathrm{C}$-terminal sides of position 0 form the ascending $\alpha$-helical $(-8$ to -1$)$ and descending random coil $(+1$ to +6$)$ components of the loop,

Glutamate receptor channels of the AMPA subtype exist as $\mathrm{Ca}^{2+}$-permeable and $\mathrm{Ca}^{2+}$-impermeable variants in functionally different sets of synapses (Geiger et al., 1995; Dingledine et al., 1999; Liu and Cull-Candy, 2000). $\mathrm{Ca}^{2+}$ permeability in these channels is defined by the identity of a single amino acid residue at the Q/R site located in the pore-lining M2 segment: glutamine renders channels permeable to $\mathrm{Ca}^{2+}$, whereas arginine prevents the permeation of $\mathrm{Ca}^{2+}$ (Hume et al., 1991; Verdoorn et al., 1991; Burnashev et al., 1992). Additionally, the Q/R site defines at least three more permeation properties of the pore. Channels containing only glutamine at the $\mathrm{Q} / \mathrm{R}$ site are blocked in a strongly voltage-dependent fashion by polyamines present in the cytoplasm of cells (Bowie and Mayer, 1995; Donevan and Rogawski, 1995; Isa et al., 1995; Kamboj et al., 1995; Koh et al., 1995), they exhibit single-channel conductances in the range of 7-8 pS (Swanson et al., 1996, 1997), and they are impermeable to anions (Burnashev et al., 1996). In contrast, channels containing only arginine at the $\mathrm{Q} / \mathrm{R}$ site are insensitive to polyamine block, have single channel conductances of $\sim 300 \mathrm{fS}$, and are slightly permeable to anions. Heteromeric channels containing both glutamine and arginine show intermediate functional profiles with a dominating contribution of the arginine residue (Dingledine et al., 1999).

Received Oct. 11, 2000; revised March 10, 2001; accepted March 22, 2001.

This work was funded by Grant SFB 317/B9 of the Deutsche Forschungsgemeinschaft to P.H.S. We thank Dr. Johannes Mosbacher for measuring fast desensitization in GluR-D $\mathrm{D}_{\mathrm{i}} \mathrm{S}$ channels, Dr. Lonnie P. Wollmuth for critically reading this manuscript, Nicole Bender and Bernhard Sakmann for help with mutagenesis in an early stage of the project, and Annette Herold for DNA sequencing.

Correspondence should be addressed to Dr. Thomas Kuner, Abteilung Molekulare Neurobiologie, Max-Planck Institut für Medizinische Forschung, Jahnstrasse 29, 69120 Heidelberg, Germany. E-mail: kuner@mpimf-heidelberg.mpg.de.

Copyright (C) 2001 Society for Neuroscience $0270-6474 / 01 / 214162-11 \$ 15.00 / 0$ respectively. Substitution of a glycine residue at position +2 with alanine strongly decreased the permeability of organic cations, indicating that position +2 contributes to the narrow constriction. The anionic 2-sulfonatoethyl-methanethiosufonate reacted with a cysteine at position 0 only from the external side and with cysteines at positions +1 to +4 only from the cytoplasmic side. These results suggest that charge selectivity occurs external to the constriction $(+2)$ and possibly involves interactions of ions with the negative electrostatic potential created by the dipole of the $\alpha$-helix formed by the ascending limb of the loop.

Key words: glutamate receptor; AMPA; narrow constriction; pore loop; SCAM; organic cations; polyamines
Therefore, a single amino acid residue at the $\mathrm{Q} / \mathrm{R}$ site controls four fundamental ionic properties of the AMPA receptor (AMPAR) channel pore, and, consequently, the function that these channels perform in the synapse. Despite the early discovery of this critical amino acid residue (Hume et al., 1991; Verdoorn et al., 1991), the structural environment of the $\mathrm{Q} / \mathrm{R}$ site and its relation to the overall structural design of the M2 segment remained poorly characterized.

AMPAR channels form large pores with a diameter of the narrow constriction estimated to be $0.78 \mathrm{~nm}$ (Burnashev et al., 1996). Interestingly, the diameter of the constriction is not affected by the identity of the residue at the Q/R site (Burnashev et al., 1996). In addition to the $\mathrm{Q} / \mathrm{R}$ site, an aspartate residue four positions downstream has been shown to play a prominent role in defining permeation properties of the AMPAR pore (Dingledine et al., 1992; Blaschke et al., 1993). Knowing the positioning of these residues relative to the constriction will further our understanding of the structural basis of ion permeation in AMPAR channels.

In this paper, we identify channel-lining residues of the AMPAR M2 segment using the substituted-cysteine-accessibility method (Akabas et al., 1992, 1994). Our results confirm that the AMPAR channel M2 segment forms a pore loop with the functionally critical $\mathrm{Q} / \mathrm{R}$ site located at the external entrance to the narrow constriction formed by position G589. Furthermore, we show that charge selectivity occurs within a small region external to the constriction, possibly involving an electrical potential created by the dipole of the $\alpha$-helix formed by the ascending limb of the loop.

Parts of this work have been published previously (Kuner et al., 1997). 


\section{MATERIALS AND METHODS}

\section{Materials}

The methanethiosulfonate (MTS) reagents were purchased from Toronto Research Chemicals (Ontario, Canada). Enzymes were obtained from New England Biolabs (Schwalbach, Germany), Boehringer Mannheim (Mannheim, Germany), Stratagene (Heidelberg, Germany), or Promega (Madison, WI). All other chemicals were purchased from either Sigma Chemicals (St. Louis, MO) or Merck (Darmstadt, Germany).

\section{Molecular biology}

All experiments were performed using expression constructs of AMPAR subunits optimized for expression in Xenopus oocytes. The coding region of the GluR- $\mathrm{D}_{\mathrm{i}}$ was subcloned into a vector derived from pSP6 (Kuner and Schoepfer, 1996); noncoding regions were removed. Two silent restriction sites ( $A p a \mathrm{I}, B s p \mathrm{EI})$ flanking the $\mathrm{M} 2$ region were introduced by PCR-based methods (Ausubel et al., 1995). Mutants were generated by PCR replacing the $105 \mathrm{bp}$ fragment defined by ApaI and BspEI. All constructs were sequenced over the entire length of the replaced fragment. Capped cRNA was transcribed with SP6 RNA polymerase and injected into Xenopus laevis oocytes as described (Kuner and Schoepfer, 1996). In some instances, wild-type cRNA was coinjected with mutant cRNA to improve current yields.

\section{Electrophysiology}

Whole-cell recordings were performed on a two-microelectrode voltageclamp system modified for automatic execution of recording protocols and solution exchange (Kuner and Schoepfer, 1996) (EggWorks, NPI Electronics, Tamm, Germany). The external solution consisted of (in $\mathrm{mm}$ ): $115 \mathrm{Na}, 2.5 \mathrm{KCl}, 1.8 \mathrm{Ca}^{2+}$, and $10 \mathrm{HEPES}$. Solutions were adjusted to $\mathrm{pH} 7.5$ with $\mathrm{NaOH}$. All drugs were applied with the bath solution.

Currents in oocyte patches were recorded with the patch-clamp technique (Hamill et al., 1981) using an EPC-9 amplifier with PULSE software (HEKA Electronics GmbH, Lambrecht, Germany). Solutions were applied using a Piezo-driven double-barrel application system (Colquhoun et al., 1992).

Giant inside-out patches were excised from Xenopus oocytes as described (Kuner et al., 1996). Symmetrical potassium solution contained (in mM): $100 \mathrm{KCl}, 10 \mathrm{HEPES}, 10$ EGTA (external), or 10 EDTA (internal), $\mathrm{pH}$ adjusted to 7.2 with $\mathrm{KOH}$. Data were low-pass-filtered at $100 \mathrm{~Hz}$ and digitized at $500 \mathrm{~Hz}$. Pipettes were pulled from borosilicate glass and had resistances of $300-500 \mathrm{k} \Omega$ when measured in potassium solution.

Outside-out patches were excised from Xenopus oocytes. The external solution was identical to that used for two-microelectrode voltage-clamp recordings. Pipettes were filled with a potassium solution and had resistances of $2-10 \mathrm{M} \Omega$.

\section{Substituted-cysteine-accessibility method}

Mutant channels were probed from the extracellular and cytoplasmic sides of the membrane with three differently sized methanethiosulfonate (MTS) derivatives: 2-aminoethyl-methanethiosufonate (MTSEA, positive charge), 2-trimethylammonioethyl-methanethiosufonate (MTSET, positive charge), and 2-sulfonatoethyl-methanethiosufonate (MTSES, negative charge). These reagents can covalently link their charged -S$\mathrm{CH}_{2}-\mathrm{CH}_{2}-\mathrm{R}$ groups to the thiolate of cysteines exposed to the wateraccessible surface of the channel. We assume that covalent modification of cysteine positioned in a narrow region of the channel persistently alters current flow. The accessibility of substituted cysteines to reaction with the MTS reagents was assayed as a persistent change of the glutamate-activated current and current rectification after exposure to MTS reagents in the presence of glutamate. MTS reagents were added to the solution at 1-3 mM (MTSEA), 1-2 mM (MTSET), and $10 \mathrm{~mm}$ (MTSES). Solutions either were prepared immediately before the experiment or kept on ice (maximally $2 \mathrm{hr}$ ) before use.

\section{Experimental protocols and data analysis}

Probing cysteine substitution mutants from the extracellular side. Oocytes were held at $-70 \mathrm{mV}$, and the baseline current amplitude $\left(I_{\text {pre }}\right)$ was established by three consecutive applications of kainate $(300 \mu \mathrm{M})$ for 60 sec. Voltage ramps $(-120$ to $+40 \mathrm{mV}$ in $2 \mathrm{sec})$ were applied before and at the end of kainate application. Immediately after the third application, solution was switched to solution containing MTS reagents for $120 \mathrm{sec}$ in the continued presence of kainate. After exposure to MTS reagents, the current amplitude $\left(I_{\text {post }}\right)$ was determined with three kainate applications.
Individual applications were separated by $120 \mathrm{sec}$ washes. Currents before and after application of the reagents were averaged, and percentage change was calculated as $\%_{\text {change }}=\left(1-I_{\text {post }} / I_{\text {pre }}\right) * 100$. Current amplitudes were corrected for rundown when appropriate.

Probing cysteine substitution mutants from the cytoplasmic side. Kainate $(100 \mu \mathrm{M})$ and cyclothiazide $(50 \mu \mathrm{M})$ were present in the pipette, and voltage ramps were applied from -110 to $110 \mathrm{mV}$ (within $2 \mathrm{sec}$ ). The leak currents were in the range of $20-50 \mathrm{pA}$ at $100 \mathrm{mV}(\sim 2-5 \mathrm{G} \Omega)$ as determined from patches containing no channels or after complete block of the current by MTS reagents. The baseline current amplitude was established by $6-12$ consecutive ramps at an interval of 5 sec. MTS reagents were applied for $60 \mathrm{sec}$, and ramps were applied continuously every $10 \mathrm{sec}$. Voltage ramps (3-20) were applied after removal of MTS reagents. Data were analyzed using Igor Pro (WaveMetrics, Lake Oswego, OR). The current amplitude at $100 \mathrm{mV}$ was determined and plotted against time. When required, currents were corrected for rundown. Percentage inhibition was calculated as $\%_{\text {inh }}=\left(1-I_{\text {post }} / I_{\text {pre }}\right) * 100$.

Apparent reaction rates. Reaction rates were determined by fitting an exponential function to the decay of the current in the presence of the reagents (Beck et al., 1999). To determine rate constants for reactions of MTS reagents applied from the extracellular side, the decay of the inward current could be fitted directly, because MTS reagents caused only a weak open-channel block of GluR- $D_{i}$ channels with the concentrations used here. In contrast, MTS reagents block GluR-D $\mathrm{D}_{\mathrm{i}} \mathrm{S}$ channels reversibly in a voltage-dependent manner when applied from the cytoplasmic side. Therefore, rate constants for reaction from the cytoplasmic side could not be determined from the decay of outward currents but were inferred from the decay of the inward current, which is only weakly blocked by the reagents (see Fig. 1). To accurately determine reaction rates, low concentrations $(20-200 \mu \mathrm{M})$ of the reagents were used.

Permeability of organic cations. The following organic cations were used to probe the size of the narrow constriction: dimethylammonium (DMA), trimethylammonium (TriMA), tetramethylammonium (TMA), tetraethylammonium (TEA), and tetrapropylammonium (TPA). External solutions contained $150 \mathrm{~mm}$ of the organic cation and $10 \mathrm{mM}$ HEPES (TMA, TEA, TPA) or $10 \mathrm{~mm}$ histidine (DMA, DEA, TriMA) adjusted to $\mathrm{pH} 7.2$ with the respective hydroxide of the organic cation or $\mathrm{HCl}$, respectively. The internal solution consisted of (in mM): $150 \mathrm{NaCl}, 10$ HEPES, and 10 EGTA, adjusted to $\mathrm{pH} 7.2$ with $\mathrm{NaOH}$. Molecular dimensions are taken from Villarroel et al. (19950 and Burnashev et al. (1996) and are summarized in Table 3. The dimensions of TPA were taken from a simulated model of TPA using SwissPdbViewer (GlaxoWellcome). Liquid junction potentials are listed in Table 3. Permeability ratios were calculated from the Goldmann-Hodgkin-Katz equation for cation-selective channels: $P_{\mathrm{x}} / P_{\mathrm{Na}}=\left(\left[\mathrm{Na}^{+}\right]_{\mathrm{i}} /\left[\mathrm{X}^{+}\right]_{\mathrm{o}}\right) \exp \left(F V_{\mathrm{rev}} / R T\right)$, where $V_{\text {rev }}$ is the measured reversal potential, $\left[\mathrm{Na}^{+}\right]_{\mathrm{i}}$ is the concentration of $\mathrm{Na}^{+}$in the pipette, $\left[\mathrm{X}^{+}\right]_{\mathrm{o}}$ is the concentration of the organic cation applied to the patch, and $F, R$, and $T$ have their usual thermodynamic meanings. Assuming a simple hydrodynamic model (Dwyer et al., 1980), the permeability of an organic cation is related to the mean diameter of the pore according to the equation: $P_{\mathrm{X}}=k\left(1-\left(d_{\text {organid }} / d_{\text {pore }}\right)\right)^{2}$.

Dose-response analysis. Peak current responses to 1, 10, 30, 100, and $300 \mu \mathrm{M}$ kainate were recorded at a holding potential of $-70 \mathrm{mV}$ and plotted against the kainate concentration. The $\mathrm{EC}_{50}$ for kainate was determined from fits of the Hill equation to the data.

Permeability to $\mathrm{Ca}^{2+}$. Outside-out patches were held in symmetric 100 $\mathrm{mm} \mathrm{K}{ }^{+}$solution and briefly exposed to $100 \mathrm{~mm} \mathrm{Ca}^{2+}$ external solution in the presence of glutamate at membrane potentials ranging from -50 to +100 in increments of $10 \mathrm{mV}$. The current-voltage $(I-V)$ relation was constructed from the peak currents corrected for leak, and a polynomial function was fitted to the data. The reversal potential was determined from the intersection of the fit with the abscissa corrected for the junction potential.

Fast desensitization. Glutamate was applied to outside-out patches using a fast piezo-driven device. Desensitization time constants were determined from exponential fits to the decaying phase of the current in the presence of glutamate, as described in Mosbacher et al. (1994).

Statistical analysis. One-way ANOVA was performed for each of the six different experimental groups (extracellular MTSEA, extracellular MTSET, extracellular MTSES, cytoplasmic MTSEA, cytoplasmic MTSET, cytoplasmic MTSES), and significance levels were calculated with the Newman-Keuls multiple comparison test using the GB-STAT software (Dynamic Microsystems, Silver Spring, MD). 

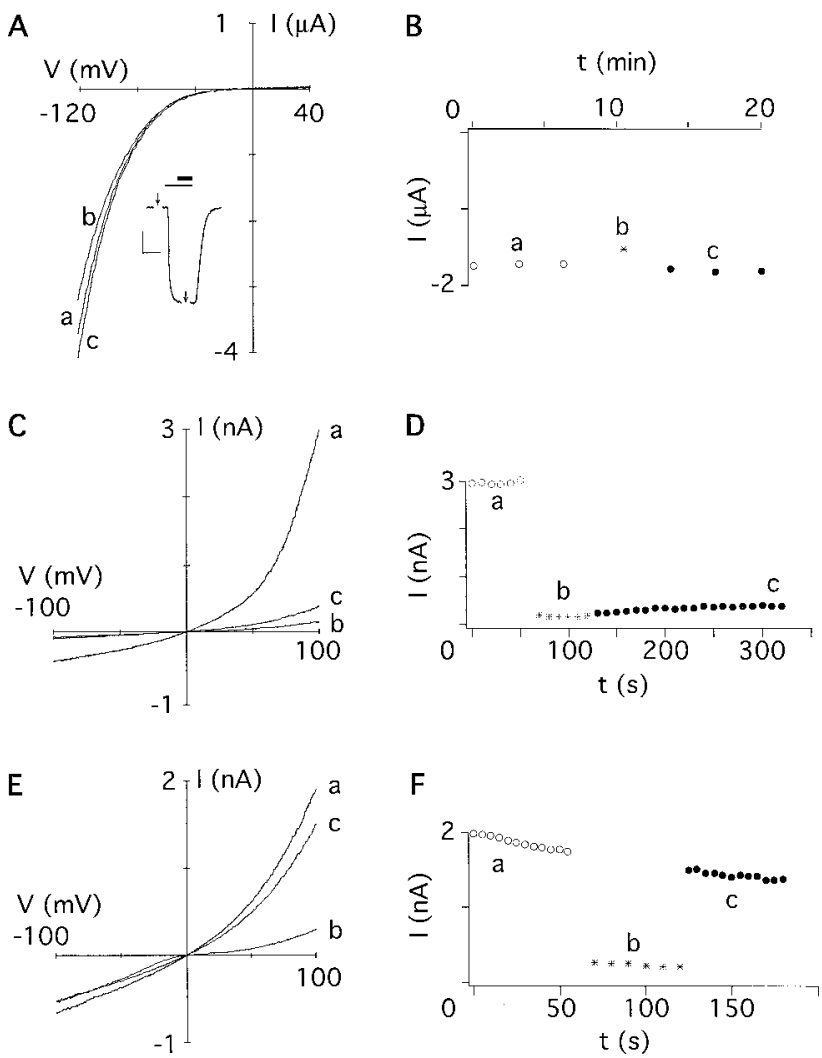

Figure 1. Effects of MTS reagents on GluR-D $\mathrm{D}_{\mathrm{i}}$ wild-type channels. $A, I-V$ relations recorded from an oocyte expressing homomeric GluR-D $D_{i}$ subunits before $(a)$, during $(b)$, and after $(c)$ application of 2 mM MTSET from the extracellular side. Currents were elicited with $300 \mu \mathrm{M}$ kainate (inset, thin bar); MTSET was applied for 1 min (inset, thick bar). Applications of control and test voltage ramps $(-120$ to $+40 \mathrm{mV})$ are marked with arrows. Calibration (inset): $100 \mathrm{nA}, 120 \mathrm{sec}$. $B$, Current amplitudes extracted from the experiment shown in $A$ at $-100 \mathrm{mV}$ plotted against the time. Kainate was applied three times before $(\bigcirc)$ and after $(\bullet)$ exposure to MTSET (asterisk). Small letters denote amplitudes extracted from the respective $I-V$ relations shown in $A$. $C, I-V$ relations recorded from a giant inside-out patch containing GluR-D $\mathrm{D}_{\mathrm{i}}$ channels subunits before $(a)$, during $(b)$, and after (c) application of $2 \mathrm{~mm}$ MTSET from the cytoplasmic side. Voltage ramps were applied in the continuous presence of kainate in the pipette. $D$, Current amplitudes extracted from the experiment shown in $C$ at $+100 \mathrm{mV}$ plotted against the time. Six ramps were applied before $(\bigcirc)$, 6 were applied during (asterisks), and 20 were applied after $(\bullet)$ exposure to MTSET. $E$, As in $C$, but the patch containing GluR-D $\mathrm{D}_{\mathrm{i}}$ channels with the native cysteine at position +3 was substituted by serine $\left(D_{i} S\right)$. Interestingly, the block by cytoplasmically applied MTSET was slightly voltage dependent, showing a current rectification reminiscent of polyamine block $(b) . F$, As in $D$, but the patch contained GluR-D $\mathrm{D}_{\mathrm{i}} \mathrm{S}$ channels. Voltage ramps were applied 12 times before $(\bullet)$ and 12 times after $(\bigcirc)$ exposure to MTSET (asterisks). The rundown of the current amplitude is within the range typically found for GluR- $\mathrm{D}_{\mathrm{i}}$ wild-type and cysteine-substituted channels.

\section{RESULTS}

\section{Effects of MTS reagents on GluR- $D_{i}$ wild-type channels}

Figure $1 A$ shows kainate-activated currents recorded from a Xenopus oocyte expressing homomeric GluR-D $\mathrm{D}_{\mathrm{i}}$ channels. The inset illustrates a current-response elicited by kainate at a membrane potential of $-70 \mathrm{mV}$, with arrows indicating the application of voltage ramps. The $I-V$ relations show a strong inward rectification caused by a voltage-dependent block of the channels by polyamines present in the oocyte. To probe for the presence of reactive cysteine residues in the conduction pore, channels were exposed to MTS reagents (MTSET, MTSEA, MTSES; see Materials and Methods). The effect of these reagents on channel function was assessed from persistent changes of the current amplitudes. Figure $1 B$ shows a plot of current amplitudes isolated at $-100 \mathrm{mV}$ as a function of time. Kainate was applied three times to establish the baseline $(a)$, inward currents were weakly blocked by MTSET $(b)$, and after washout of the reagent the current amplitudes returned to baseline $(c)$. In four independent experiments, the relative change of the current amplitude ( $I_{\text {post }} /$ $I_{\text {pre }}$ ) was $1 \pm 4 \%$, and the current rectification remained unchanged. Similar results were obtained with MTSEA ( $-5 \pm 7 \%$; $n=5)$ and MTSES $(-3 \pm 6 \% ; n=3)$, none of which persistently changed the current amplitude or current rectification. These results suggest that the extracellular part of the pore of GluR-D wild-type channels does not contain functionally relevant porelining cysteine residues.

Figure $1 C$ shows $I-V$ relations recorded from a giant inside-out patch containing GluR- $\mathrm{D}_{\mathrm{i}}$ channels. In symmetrical divalent-free potassium solution, in the nominal absence of polyamines on the cytoplasmic side, GluR-D $\mathrm{D}_{\mathrm{i}}$-mediated currents were outwardly rectifying (Fig. 1C, a). Current amplitudes extracted at $+100 \mathrm{mV}$ and plotted against the time are shown in Figure $1 D$. After the baseline was established $(a)$, application of MTSET from the cytoplasmic side resulted in a strong block of both inward and outward currents $(b)$. Surprisingly, the current remained persistently blocked (93 $\pm 3 \% ; n=4)$ after washout of MTSET $(c)$. Cytoplasmic application of MTSEA yielded a similar result (96 \pm $5 \% ; n=6)$. Exposure of the channels to MTSES persistently blocked the current by $82 \pm 11 \%(n=4)$, but the onset of block was markedly slower in comparison to MTSET and MTSEA (see below). These results suggest that MTS reagents applied to the cytoplasmic side of homomeric GluR- $\mathrm{D}_{\mathrm{i}}$ channels reacted with a pore-lining cysteine residue, which was not accessible, however, from the extracellular side.

The GluR-D $\mathrm{D}_{\mathrm{i}}$ subunit contains cysteine at position +3 (C590) of the M2 segment, a residue present only in AMPAR subunits but in none of the other GluR subunits (Fig. 2). We substituted the cysteine residue with serine, an amino acid of similar size occupying the homologous position in kainate receptors. Probing a GluR- $\mathrm{D}_{\mathrm{i}}(\mathrm{C}+3 \mathrm{~S})$ channel with MTSET from the cytoplasmic side revealed that this channel was rendered insensitive to persistent modification by MTSET (Fig. $1 E, F$ ). After the baseline was established $(a)$, application of MTSET strongly blocked the channel $(b)$, and the current amplitude returned to baseline during washout $(c)$. From this result we infer that the native cysteine residue at position +3 mediates inhibition of wild-type GluR- $D_{i}$ and hence is exposed in the lumen of the channel. Consequently, GluR-D $D_{i}$ wild-type subunits cannot be used to identify pore-lining residues of the M2 segment using the substituted-cysteine-accessibility method. However, the serinesubstituted GluR-D $(\mathrm{C}+3 \mathrm{~S})$ subunit could be used as a host for cysteine substitutions, given that this mutant channel is near wild-type in its structural and functional properties. Indeed, we did not find differences in maximal whole-cell currents, $\mathrm{EC}_{50}$ of kainate, fast desensitization, permeability to $\mathrm{Ca}^{2+}$, and diameter of the pore (Table 1). Hence, GluR- $\mathrm{D}_{\mathrm{i}}(\mathrm{C}+3 \mathrm{~S})$ subunits can be used in place of wild type as a host for cysteine substitutions.

\section{Cysteine-substitutions in the GluR- $D_{i}(C+3 S)$ subunit}

Cysteine was substituted at positions -8 to +6 in the M2 segment of the GluR-D $(\mathrm{C}+3 \mathrm{~S})$ subunit, herein referred to as "DiS" subunit (Fig. 2). Most of the 14 cysteine-substituted channels 


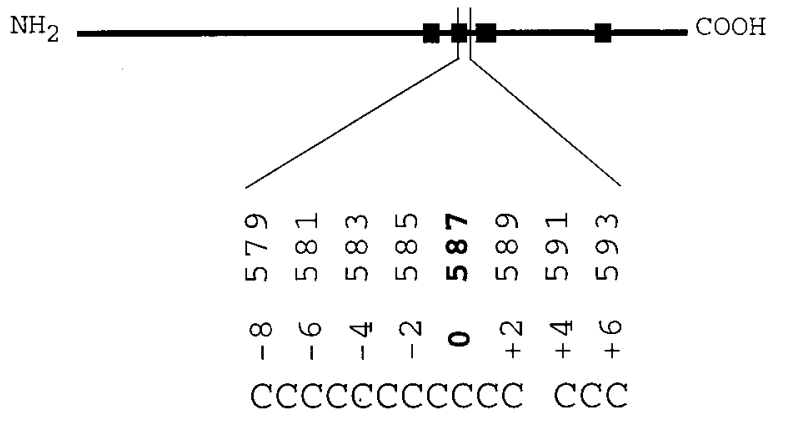

$\begin{array}{ll}\text { Dis } & \text { WFSLGAFMQQGSDIS } \\ \text { Di } & \text { WESLGAFMQQGCDIS } \\ \text { GIUR6 } & \text { WFGMGSLMQQGSELM } \\ \text { NR1 } & \text { WFSWGVLINSGIGEG } \\ \text { NR2C } & \text { WILWALVENNSVPIE }\end{array}$

Figure 2. Alignment of selected GluR M2 segments and positions where cysteine was substituted. Schematic representation of a GluR subunit (thick line) with the black boxes denoting the four hydrophobic domains. Numbers (579-593) refer to positions of the amino acid residues of the M2 segment in the mature GluR- $\mathrm{D}_{\mathrm{i}}$ subunit. To facilitate comparisons between subunits, we use a relative numbering system with negative numbers denoting positions $\mathrm{N}$ terminal to position $0(\mathrm{Q} / \mathrm{R}$ site) and positive numbers on the C-terminal site (Kuner et al., 1996). DiS refers to a subunit in which the native cysteine residue at position +3 was replaced with a serine residue. This subunit was used for cysteine substitutions at positions -8 to +6 .

Table 1. GluR-Di $(\mathrm{C}+3 \mathrm{~S})$ is functionally equivalent to wild type

\begin{tabular}{|c|c|c|}
\hline & GluR-Di & $\begin{array}{l}\text { GluR- } \\
\mathrm{Di}(\mathrm{C}+3 \mathrm{~S})\end{array}$ \\
\hline Current at $-100 \mathrm{mV}(\mu \mathrm{A})$ & $-10 \pm 1.5(5)$ & $-11 \pm 3.5(5)$ \\
\hline $\mathrm{EC}_{50}$ of kainate $(\mu \mathrm{M})^{a, b}$ & $68 \pm 30(3)$ & $49 \pm 32(3)$ \\
\hline Desensitization time constant ${ }^{a}(\mathrm{msec})$ & $3.6 \pm 0.6(10)^{c}$ & $3.0 \pm 1.1(3)$ \\
\hline $\mathrm{Ca}^{2+}$ reversal potential $^{a}(\mathrm{mV})$ & $16.8 \pm 2.1(3)$ & $18.4 \pm 3.2(3)$ \\
\hline Pore diameter $(\mathrm{nm})$ & $0.75 \pm 0.04 *$ & $0.75 \pm 0.05^{*}$ \\
\hline
\end{tabular}

${ }^{a}$ See Materials and Methods.

${ }^{b}$ Hill coefficients close to unity.

${ }^{c}$ Values taken from Mosbacher et al. (1994).

*SD derived from fit to the hydrodynamic equation (see Materials and Methods).

yielded current amplitudes comparable with wild-type; only four mutations, $\mathrm{W}-8 \mathrm{C}, \mathrm{F}-7 \mathrm{C}, \mathrm{Q}+1 \mathrm{C}$, and $\mathrm{G}+2 \mathrm{C}$, gave rise to maximal current amplitudes smaller than $-0.5 \mu \mathrm{A}$ at $-100 \mathrm{mV}$ (Table 2). Within the narrow region of the channel, engineered cysteine residues of adjacent subunits might be positioned close to each other, possibly allowing the formation of disulfide bridges, which could interfere with the function of the channel. However, DTT treatment $(10 \mathrm{~mm} ; 5 \mathrm{~min})$ did not lead to an increase of the current amplitudes elicited by $300 \mu \mathrm{M}$ kainate, suggesting that other mechanisms are responsible for the small current amplitudes produced by these mutant channels (data not shown). To avoid experimental problems associated with mutant channels yielding only small current amplitudes, mutant subunits were coexpressed with the DiS subunit at a 1:1 cRNA ratio, which in all instances increased current amplitudes significantly (Table 2, lower section). Furthermore, assuming that heteromeric channels were formed, this approach allowed us to titrate the average number of cysteine residues present at a defined position in the pore of a population of channels by coinjecting different ratios of

\begin{tabular}{lc}
\hline Table 2. Current expression of cysteine-substituted channels \\
& $\mu$ A at $-100 \mathrm{mV}$ \\
Position & {$[$ mean \pm SD $(n=5)]$} \\
\hline C+3S ("DiS") & $a$ \\
W-8C & $a$ \\
F-7C & $-11.0 \pm 3.4 \pm 3.3$ \\
S-6C & $-6.3 \pm 3.8$ \\
L-5C & $-11.3 \pm 2.6$ \\
G-4C & $-10.6 \pm 3.7$ \\
A-3C & $-4.5 \pm 1.3$ \\
F-2C & $-10.0 \pm 1.6$ \\
M-1C & $-13.0 \pm 1.6$ \\
Q0C & $a$ \\
Q+1C & $-0.5 \pm 0.1$ \\
G+2C & $-10.1 \pm 1.5$ \\
C+3 & $-6.2 \pm 0.7$ \\
D+4C & $-6.2 \pm 3.3$ \\
I+5C & $-10.8 \pm 2.3$ \\
S+6C & $-4.5 \pm 2.5$ \\
W-8C + DiS 1:1 & $-4.7 \pm 1.0$ \\
F-7C + DiS 1:1 & $-9.5 \pm 2.0$ \\
Q+1C+DiS 1:1 & $-5.0 \pm 0.9$ \\
G+2C+DiS 1:1 &
\end{tabular}

${ }^{a}$ No currents detectable.

cRNAs coding for the DiS subunit and the cysteine-substituted subunit.

\section{Effects of MTS reagents applied from the extracellular side}

Application of MTS reagents on the extracellular side of the cysteine-substituted channels revealed persistent changes of the current amplitude in 4 of 15 positions tested (Figs. 3, 4; summarized in Fig. 5). Channels with cysteine at position $0(=\mathrm{Q} / \mathrm{R}$ site $)$ were strongly blocked by $1 \mathrm{~mm}$ MTSET (Fig. $3 A$, inset). After washout of MTSET, a slow recovery from the block was observed (Fig. 3B), a behavior different from that of the other mutants tested (see below). However, the current amplitudes reached a steady-state level within $\sim 20-30 \mathrm{~min}$, indicating that covalent modification of the cysteine had occurred. Homomeric DiS $(\mathrm{G}+$ 2C) channels were not persistently modified by MTSET (Fig. 3C, $b$ ); only when coexpressed with DiS channels did a persistent block of the current develop (Fig. 3D, $b$ ), suggesting that the two subunits formed heteromeric channels. Interestingly, currents passed by $\mathrm{G}+2 \mathrm{C}$ channels were only weakly rectifying (Fig. $3 C$ ), suggesting that polyamine block was abolished in homomeric $\mathrm{G}+$ $2 \mathrm{C}$ channels. Coexpression of $\mathrm{G}+2 \mathrm{C}$ with DiS channels resulted in an increased current rectification, suggesting a partial restoration of polyamine block in channels likely to contain both cysteine and glycine at position +2 (Fig. $3 D, a)$. Currents mediated by DiS $(D+4 C)$ channels were persistently decreased after exposure to MTSET (Fig. 3E, F). In summary, 3 of 15 positions tested were accessible to covalent modification by extracellularly applied MTSET: Q0C, G+2C, and D+4C (Fig. $5 A$ ).

A similar pattern was found using the smaller MTSEA (Fig. $5 B)$. Unlike MTSET, MTSEA revealed an additional residue accessible to covalent modification. When DiS(Q+1C)/DiS channels (1:1 cRNA ratio) were exposed to MTSEA, the current amplitude recorded at $-100 \mathrm{mV}$ did not change significantly (Fig. 4). However, the $I-V$ relation was less outwardly rectifying after 
A

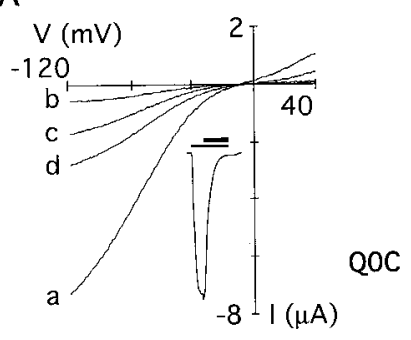

C

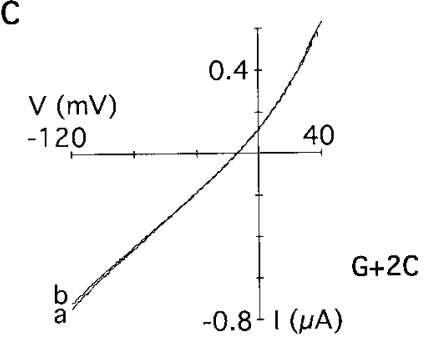

E

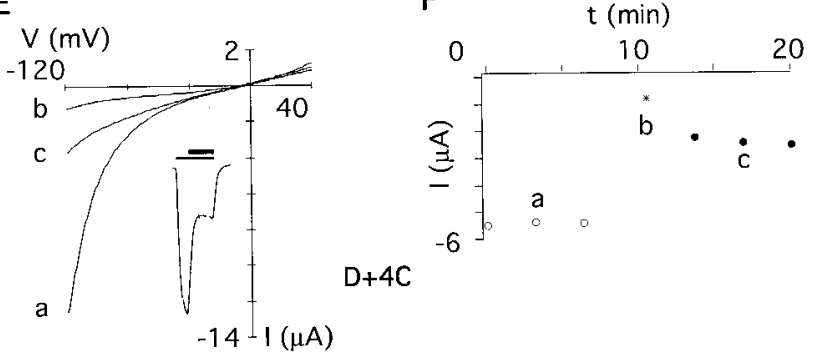

Figure 3. Extracellular application of MTSET to cysteine-substituted GluR-D $\mathrm{S}$ channels. $A, I-V$ relations recorded from an oocyte expressing homomeric GluR-D $\mathrm{S}(\mathrm{Q} 0 \mathrm{C})$ channels before $(a)$, during $(b)$, and after $(c$, d) application of $2 \mathrm{mM}$ MTSET. Currents were elicited with $300 \mu \mathrm{M}$ kainate (inset, thin bar, $90 \mathrm{sec}$ ), MTSET was applied for $60 \mathrm{sec}$ (inset, thick bar). Current shown in the inset is $4 \mu \mathrm{A}$ at $-70 \mathrm{mV}$. B. Current amplitudes of the $I-V$ relations shown in $A$ extracted at $-100 \mathrm{mV}$ plotted against the time. Kainate was applied 9 times before $(\bigcirc)$ and 13 times after (-) exposure to MTSET (asterisk). The box denotes the duration of a typical experiment as shown for all other cysteine-substituted channels. $C, I-V$ relation recorded from an oocyte expressing homomeric GluR$\mathrm{D}_{\mathrm{i}} \mathrm{S}(\mathrm{G}+2 \mathrm{C})$ channels $(a)$. The currents remained unchanged during and after exposure to $2 \mathrm{~mm}$ MTSET $(b) . D, I-V$ relation recorded from an oocyte injected with a 1:1 ratio of cRNAs encoding GluR- $\mathrm{D}_{\mathrm{i}} \mathrm{S}$ and GluR-D $\mathrm{D}_{i} \mathrm{~S}(\mathrm{G}+2 \mathrm{C})$ subunits $(a)$. In heteromeric channels, MTSET $(2 \mathrm{mM})$ persistently reduced the current amplitude $(b)$. $E$, As in $A$, but recorded from an oocyte expressing homomeric GluR- $\mathrm{D}_{\mathrm{i}} \mathrm{S}(\mathrm{D}+4 \mathrm{C})$ channels. Current shown in the inset is $4 \mu \mathrm{A}$ at $-70 \mathrm{mV}$. $F$, As in $B$, but three applications of kainate before and after exposure to MTSET.

covalent modification (Fig. 4A), suggesting an interference of the modified cysteine with polyamine block. Reaction of the smaller MTSEA, but not the larger MTSET, with the cysteine at position +1 may suggest that steric constraints limit the accessibility of the side chain at position +1 .

The negatively charged MTSES reacted only with DiS(Q0C) mutant channels (Fig. 5C). In summary, all three reagents reacted with a cysteine at position 0 , but only the positively charged MTSEA and MTSET reacted with residues on the C-terminal side of position 0 . These results suggest that anions can reach as far down into the pore as position 0 , but a charge selectivity barrier may prevent the negatively charged MTSES from reacting with $\mathrm{G}+2 \mathrm{C}$ and $\mathrm{D}+4 \mathrm{C}$. Given that the size of MTSES is in between that of MTSEA and MTSET, it should have reacted with
A

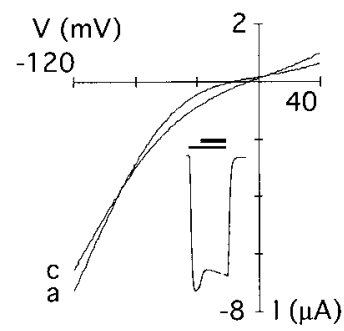

B

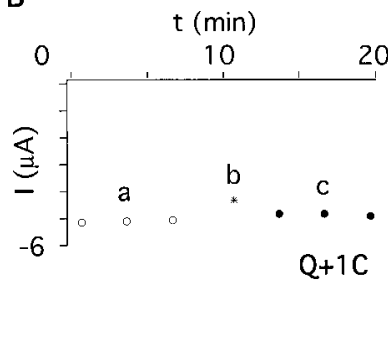

Figure 4. Extracellular application of MTSEA to GluR-D $\mathrm{S}(\mathrm{Q}+1 \mathrm{C})$ channels. $A$, Currents recorded from an oocyte expressing homomeric GluR- $\mathrm{D}_{\mathrm{i}} \mathrm{S}(\mathrm{Q}+1 \mathrm{C})$ channels. Current shown in the inset is $2.5 \mu \mathrm{A}$ at -70 $\mathrm{mV}$. For clarity, curve $b$ has been removed. B, Current amplitudes extracted at $-100 \mathrm{mV}$ plotted against the time.

cysteine residues at these positions if steric constraints were the limiting factor.

In conclusion, channels with substitutions $\mathrm{Q} 0 \mathrm{C}, \mathrm{Q}+1 \mathrm{C}, \mathrm{G}+2 \mathrm{C}$, and $\mathrm{D}+4 \mathrm{C}$ exhibited persistent changes in current amplitudes after extracellular application of MTS reagents and hence line the pore of GluR-D $\mathrm{D}_{\mathrm{i}}$ receptor channels.

\section{Effects of MTS reagents applied from the cytoplasmic side}

About half of the mutant channels were susceptible to covalent modification when the MTS reagents were applied from the cytoplasmic side. A cysteine at position 0 , which was accessible to all three reagents from the extracellular side, was modified only by cytoplasmically added MTSEA, not by MTSET or MTSES (Fig. 6A,B; summary in Fig. 7). MTSEA applied after exposure to MTSET or MTSES resulted in irreversible inhibition, suggesting that MTSET and MTSES did not react silently with the cysteine (data not shown). The possibility remains that the neutral form of MTSEA, which can passively diff use through membranes (Holmgren et al., 1996), reacted from the extracellular side. However, $10 \mathrm{~mm}$ cysteine in the pipette did not affect the reaction of MTSEA with the cysteine at position 0, suggesting that MTSEA could access it directly through the channel. These observations may suggest that the larger MTSET could not react because of steric constraints, possibly imposed by a narrow constriction, and that the negatively charged MTSES could not cross the selectivity filter. Substituted cysteines on the C-terminal side of position $0, \mathrm{Q}+1 \mathrm{C}, \mathrm{G}+2 \mathrm{C}$, and $\mathrm{D}+4 \mathrm{C}$ were modified by all three reagents (Figs. 6, 7), suggesting a sterically and electrostatically unrestricted access pathway. Because homomeric $\mathrm{DiS}(\mathrm{Q}+$ 1C) and $\mathrm{DiS}(\mathrm{G}+2 \mathrm{C})$ channels yielded only low current amplitudes, these subunits were coexpressed with DiS subunits. Figure $6 C$ shows irreversible modification by MTSET of cysteine in channels formed by $\mathrm{DiS}(\mathrm{G}+2 \mathrm{C})$ and DiS subunits coinjected with a 1:1 cRNA ratio. Given the heteromeric nature of this channel, this result suggests that the modification of a number of cysteine residues less than the number of subunits forming the channel is sufficient to strongly inhibit the current. A cysteine at position +4 created a change of the intrinsic current rectification and resulted in an inwardly rectifying current in the absence of polyamines and divalent ions (Fig. 6E). Exposure to MTSET produced a strong and irreversible inhibition of the current amplitudes (Fig. $6 F$ ). On the N-terminal side of position 0, only A-3C, G-4C, and F-7C were accessible to the reagents (Fig. 7). The large MTSET reacted only with cysteines at positions -7 and -4 , whereas the two smaller reagents, MTSEA and MTSES, in addition reacted 


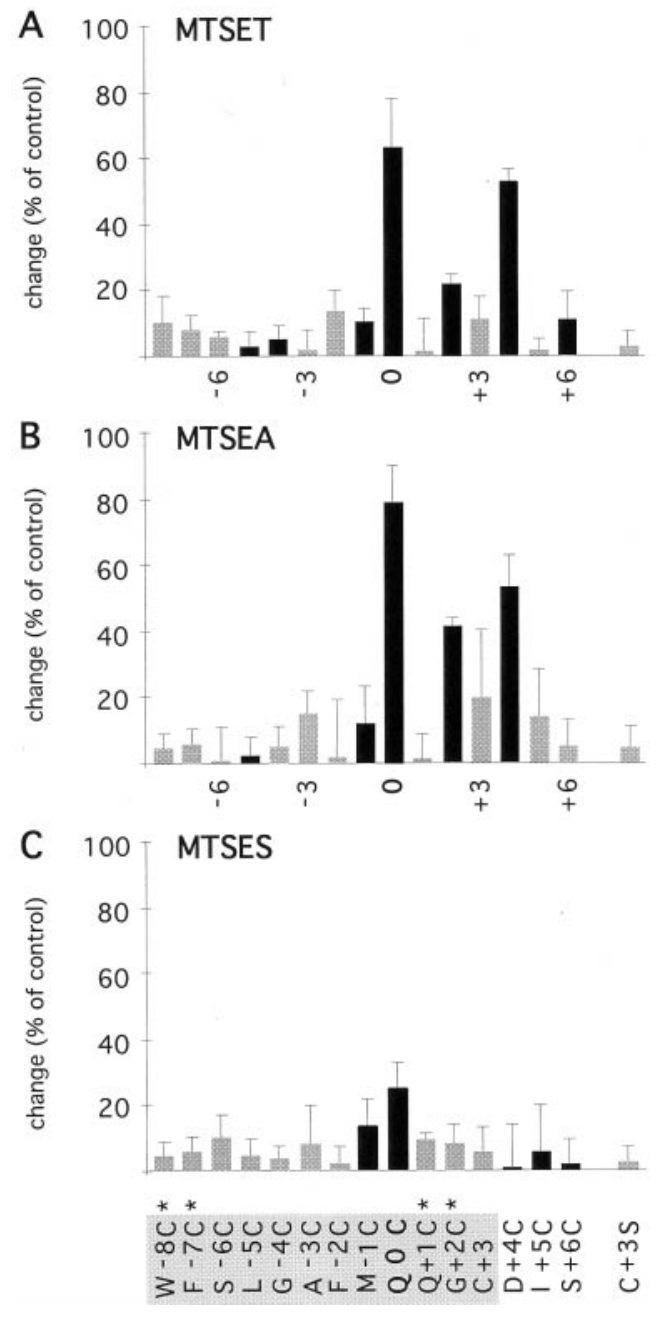

Figure 5. Effects of extracellularly applied MTS reagents on cysteinesubstituted GluR-D $\mathrm{D}_{\mathrm{i}} \mathrm{S}$ channels. $A-C$, Mean change of the current amplitude after exposure to MTSET, MTSEA, and MTSES applied from the extracellular side. Black bars denote current inhibition, and shaded bars represent current potentiation after exposure to the reagents. Shown is the mean change \pm SD of 3-10 experiments. For a binary interpretation of the data based on statistical analysis, see Figure 9. Asterisks indicate coexpression with GluR- $\mathrm{D}_{\mathrm{i}} \mathrm{S}$ subunits.

with a cysteine at position -3 . It remained unclear whether a cysteine at position -8 is exposed to the pore, because expression of W-8C subunits did not yield detectable currents (Table 2). Therefore, mutant subunits with a W-8C substitution may not form heteromeric channels when coexpressed with the DiS subunit. In summary, cysteine-substituted channels F-7C, G-4C, A-3C, Q0C, Q+1C, G+2C, D+4C, and S+6C were persistently inhibited by cytoplasmically applied MTS reagents (Fig. 7) and thus contribute part of the channel wall in GluR- $\mathrm{D}_{\mathrm{i}}$ receptor channels.

\section{Reaction rates of MTS reagents with substituted cysteines}

We determined apparent reaction rates for covalent modification of exposed cysteine residues by MTS reagents from the decay of the current in the presence of the reagents (Fig. 4, inset) (see Materials and Methods). When applied from the extracellular side, MTSET and MTSEA showed rather slow reaction rates, with no significant differences between exposed cysteines Q0C,
A

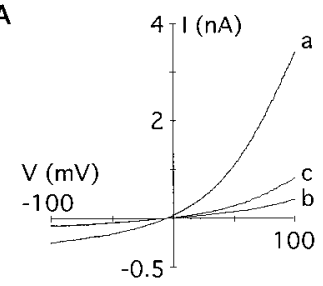

C
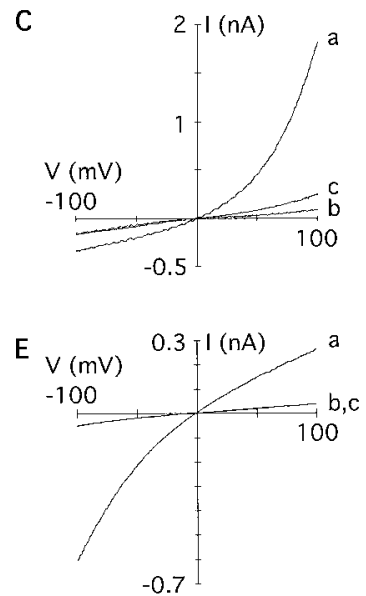

B

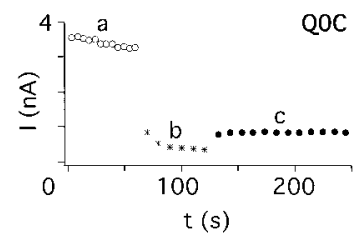

D

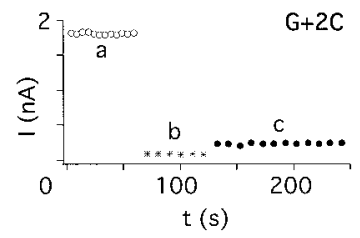

$\mathrm{F}$

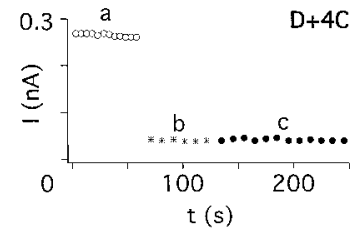

Figure 6. Cytoplasmic application of MTS reagents to cysteinesubstituted GluR-D $\mathrm{D}_{\mathrm{i}} \mathrm{S}$ channels. $A, I-V$ relations recorded from a giant inside-out patch containing homomeric GluR- $\mathrm{D}_{\mathrm{i}} \mathrm{S}(\mathrm{Q} 0 \mathrm{C})$ channels before $(a)$, during $(b)$, and after $(c)$ application of 2 mM MTSEA. Currents were elicited with $300 \mu \mathrm{M}$ kainate in the pipette. $B$, Current amplitudes extracted from the experiment shown in $A$ at $+100 \mathrm{mV}$ are plotted against the time. Kainate was applied 12 times before $(\bigcirc)$ and 12 times after $(\bullet)$ exposure to MTSEA (asterisk). Letters $a-c$ denote amplitudes extracted from the experiment shown in $A$. $C$, As in $A$, with channels containing GluR-D $\mathrm{D} S(\mathrm{G}+2 \mathrm{C})$ and GluR-D $\mathrm{D}_{\mathrm{i}} \mathrm{S}$ subunits and using MTSET instead of MTSEA. $D$, As in $B$, with experiment shown in $C$. $E$, As in $A$, with GluR-D $\mathrm{S}(\mathrm{D}+4 \mathrm{C})$ channels. The intrinsic current rectification was altered by the mutation. $F$, As in $B$, with experiment shown in $E$.

$\mathrm{G}+2 \mathrm{C}$, and D+4C (Fig. 8). Reaction of MTSES with a cysteine at position 0 was markedly slower than reaction of the positively charged MTSET and MTSEA (Fig. 8), suggesting that a negative electrostatic potential close to position 0 may limit accessibility to a negatively charged reagent. MTSET applied from the cytoplasmic side reacted up to $\sim 100$ times faster, with $\mathrm{G}+2 \mathrm{C}$ and $\mathrm{D}+4 \mathrm{C}$ showing the fastest reaction rates (Fig. 8, black bars). Similar rate constants were found for MTSEA (gray bars), although with cysteine at position +2 or +3 , MTSEA showed a faster reaction rate than MTSET, suggesting that accessibility to positions +2 and +3 may be sterically restricted. MTSES exhibited low reaction rates $\left(\sim 1-5 \mathrm{M}^{-1} \mathrm{sec}^{-1}\right)$ throughout (white bars), consistent with restricted access of anions to the narrow region of a cationselective channel. However, MTSES reacted $\sim 100 \times$ faster with a cysteine at position +4 , suggesting that this position may be located at the internal opening to the narrow region. In conclusion, the reaction rates observed for covalent modification of the substituted cysteines are in agreement with a location at the aqueous surface lining the lumen of the channel.

\section{Location of the narrow constriction}

Figure 9 summarizes the accessibility patterns in a binary fashion and compares the different reagents and relative sidedness of accessibility. A simple pattern is found for MTSES: position 0 was accessible from the external side, whereas positions flanking 0 

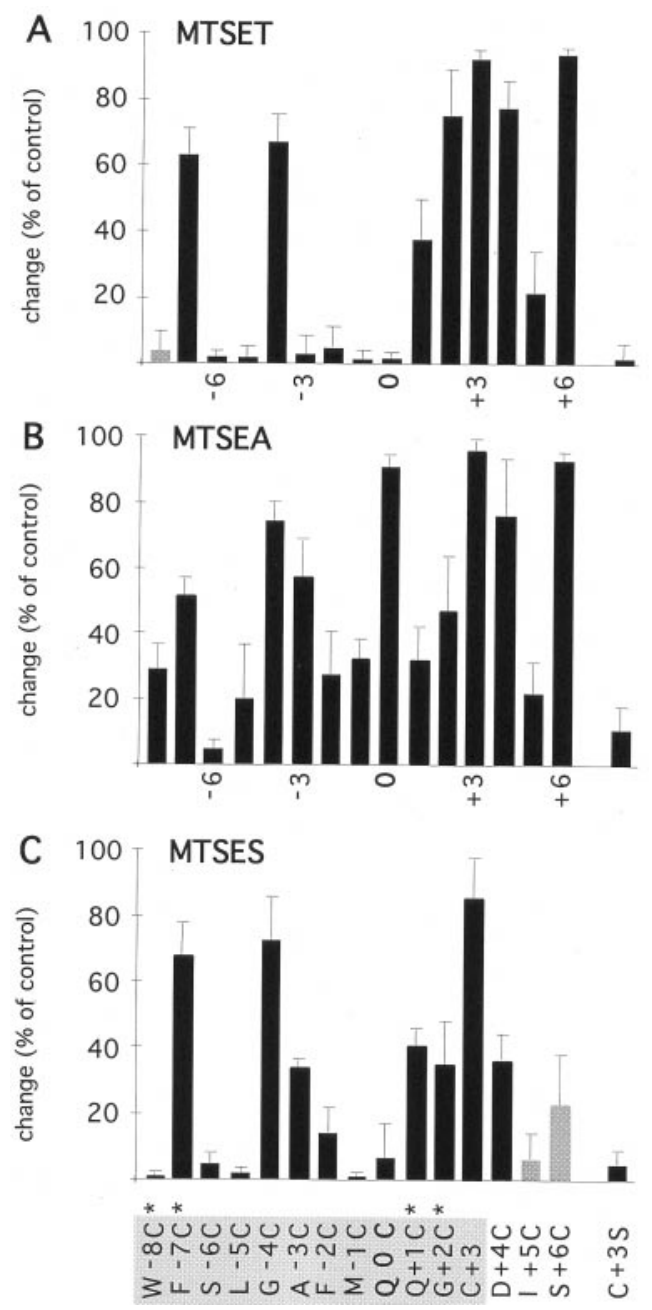

Figure 7. Effects of cytoplasmically applied MTS reagents on cysteinesubstituted GluR-D ${ }_{\mathrm{i}} \mathrm{S}$ channels. $A-C$, See legend to Figure 5.

were accessible from the internal side. Assuming that MTSES is impermeant, such a pattern is expected if the selectivity filter is located between positions -3 and 0 or 0 and +1 . A common pattern emerged for the two positively charged reagents: several positions from 0 to +4 were accessible from either side of the channel, whereas positions on the $\mathrm{C}$ - and $\mathrm{N}$-terminal sides were accessible only from the cytoplasmic side. Furthermore, covalent modification of cysteines at positions 0 to +4 had the strongest effects on current amplitudes, intrinsic rectification, and polyamine block. These findings suggested that a narrow region of the channel may form between residues 0 and +4 . To test this hypothesis further, we examined the effects of mutations in this stretch of residues on the size of the narrow constriction estimated from the permeability of organic cations relative to sodium. Residue $G+2$ seemed particularly interesting, because polyamine block was almost abolished and a cysteine at this position was inaccessible in homomeric $\mathrm{G}+2 \mathrm{C}$ channels (Fig. $3 C$ ), but accessible in channels containing both $\mathrm{G}+2 \mathrm{C}$ and $\mathrm{D}_{\mathrm{i}} \mathrm{S}$ subunits (Fig. 3D). A strong decrease in the size of the constriction by the $\mathrm{G}+2 \mathrm{C}$ substitution may explain both observations: the constriction might be too small for polyamines to bind, and MTSET could not react because of steric constraints. To create a mutant channel with only a small change in pore size compared with wild type, we substituted the glycine residue at position +2

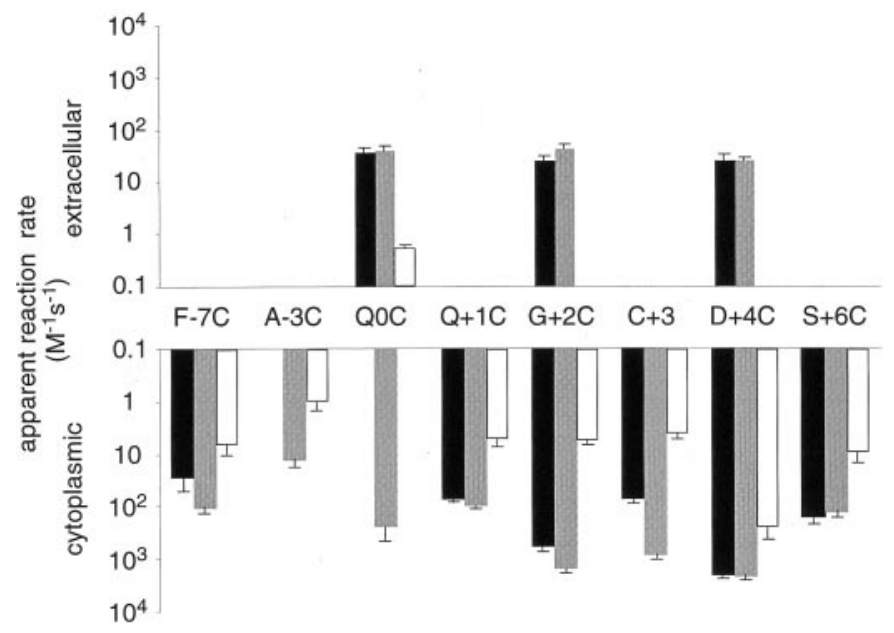

Figure 8. Apparent reaction rates of MTS reagents with substituted cysteines. Reaction rates of MTSET (black bars), MTSEA (gray bars), and MTSES (white bars). Rate constants for reactions from the extracellular side are shown in the top panel; those for reactions from the cytoplasmic side are shown in the bottom panel. Values are plotted as mean \pm SEM of three to five experiments.

with an alanine residue. Reversal potentials in bi-ionic conditions were recorded from outside-out patches containing channels assembled from DiS or DiS $(\mathrm{G}+2 \mathrm{~A})$ subunits. Figure $10 A$ shows current-responses mediated by homomeric DiS channels in outside-out patches at different voltages. The bath solution contained $100 \mathrm{~mm}$ TMA with $100 \mathrm{mM} \mathrm{Na}^{+}$in the pipette. The reversal potential under these conditions was $-32.1 \pm 3 \mathrm{mV}(n=$ 11). In $\mathrm{DiS}(\mathrm{G}+2 \mathrm{~A})$ channels (Fig. 10B), the reversal potential was strongly shifted leftward to $-75.5 \pm 2.5 \mathrm{mV}(n=4)$, suggesting that the dimensions of the narrow constriction are much smaller in DiS $(G+2 A)$ channels than in DiS channels. To quantify the change in the dimension of the pore, reversal potentials of DMA, TriMA, TEA, and TPA were determined (Table 3). The relative permeability of organic cations over the permeability of $\mathrm{Na}^{+}$was plotted against the mean diameter of the organic cations, and the data were fitted to the hydrodynamic equation (Fig. $10 C$ ) (see Materials and Methods). In this analysis, the diameter of the pore of DiS channels was $\sim 0.75 \mathrm{~nm}$, in close agreement with previous results obtained from wild-type GluR-A channels (Burnashev et al., 1996). The diameter of the pore of DiS $(\mathrm{G}+2 \mathrm{~A})$ channels was markedly smaller, measuring only $\sim 0.58 \mathrm{~nm}$. These results demonstrate that the residue at position $\mathrm{G}+2$ contributes to the narrow constriction of GluR- $\mathrm{D}_{\mathrm{i}}$ channels.

\section{DISCUSSION}

The present work elucidates the structural environment of the functionally critical Q/R site in AMPAR channels. We demonstrate that the M2 segment of the GluR-D $D_{i}$ subunit forms a pore loop originating on the cytoplasmic side of the membrane, with the $\mathrm{Q} / \mathrm{R}$ site located at its tip (position 0), and the narrow constriction formed by position +2 (Fig. 11). To infer that a residue contributes to the lining of the channel, we assume the following: first, substitution with a cysteine does not change the gross structure of the channel; second, only residues exposed in the water-filled lumen of the channel are accessible to rapid covalent modification; and third, a persistent change in channel function indicates that covalent modification of the introduced cysteine had occurred. 


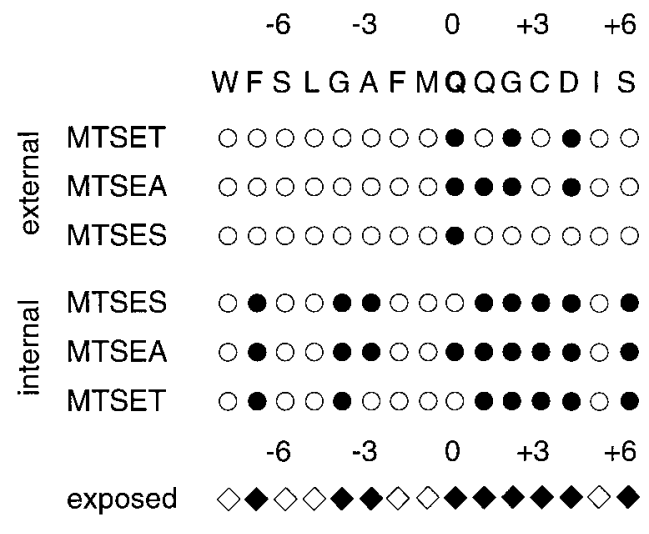

Figure 9. Binary representation of cysteine accessibility patterns: channel-lining residues of the GluR- $\mathrm{D}_{\mathrm{i}} \mathrm{M} 2$ segment. The results shown in Figures 4 and 6 were analyzed with an ANOVA test, and significance levels were calculated using the Newman-Keuls procedure. Positions at which cysteine substitution led to a persistent change of the current amplitude or current rectification are shown as filled circles, whereas positions that remained unchanged after exposure to the reagents are shown as open circles. Externally accessible positions are grouped in the top part of the Figure and internally accessible residues are shown on the bottom part. The accessibility patterns found for the three MTS reagents were translated to a pattern of exposed residues (diamonds). Numbers denote residue position relative to the position $0(\mathrm{Q} / \mathrm{R}$ site $)$.

\section{The M2 segment of the GluR- $D_{i}$ subunit forms a pore loop}

As originally shown in $\mathrm{K}$ channels (MacKinnon and Yellen, 1990), re-entrant loop segments are defined by a characteristic pattern: a residue at the tip of the loop only interacts with impermeant reagents applied from one side of the membrane, whereas flanking residues interact only with reagents applied from the opposite side. Such a pattern is present in the accessibility profile of the negatively charged MTSES (Fig. 11A), confirming that the M2 segment of the GluR- $\mathrm{D}_{\mathrm{i}}$ subunit forms a loop. The patterns found for the positively charged reagents MTSEA and MTSET are consistent with a pore loop structure as well, although the two-sided accessibility (Fig. 11B, diamonds) of several residues, which could be explained if MTSEA and MTSET permeated through the channel, complicates the interpretation of the results. Attempts to measure the permeability of the MTS reagents in bi-ionic conditions with concentrations of up to 150 mM MTS reagent failed to demonstrate permeability of the reagents, because GluR- $\mathrm{D}_{\mathrm{i}} \mathrm{S}$ channels were persistently inactivated on exposure to such high concentrations of the reagents (data not shown). On the basis of size, both MTSEA $(1 \times 0.48 \times 0.48 \mathrm{~nm})$ and MTSET $(1 \times 0.58 \times 0.58 \mathrm{~nm})$ should permeate through a pore with the smallest diameter of $0.75 \mathrm{~nm}$ and react with cysteines exposed on the opposite side of the selectivity filter. However, we found two-sided accessibility only for cysteines at the tip and at positions $+1,+2$, and +4 , but not for cysteines located farther away from the tip. One possible explanation for this discrepancy could be that the flux rate of the reagents through the channel is low and that the reagents may become rapidly diluted after passing the narrow region of the channel, resulting in concentrations too low to produce covalent modification. Alternatively, the presence of polyamines in the oocyte whole-cell experiments performed to probe extracellular accessibility may prevent permeation of the reagents but still allow reaction with the residues at or close to the narrow constriction. Interestingly, the larger MTSET showed a similar pattern of two-sided acces-
A

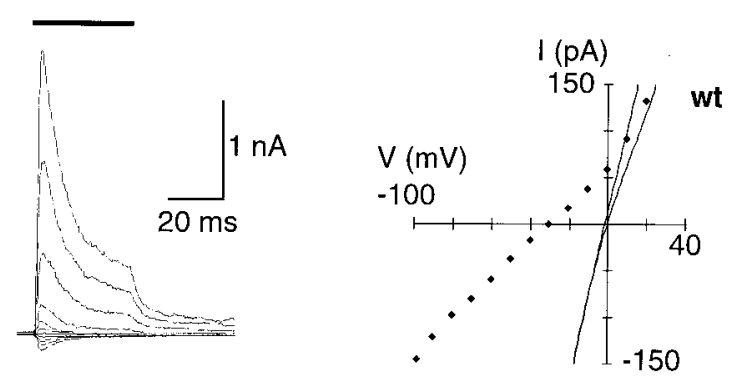

B
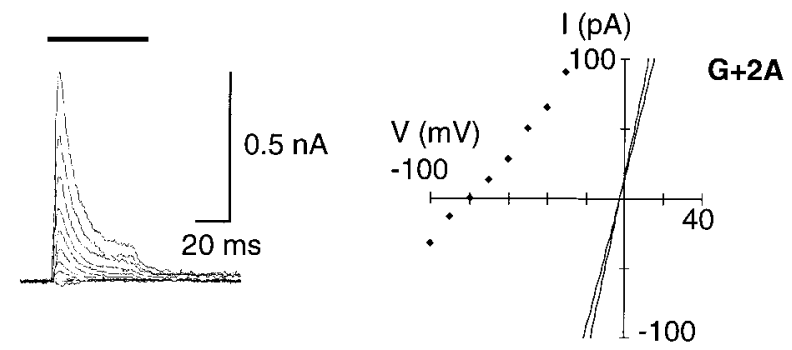

C

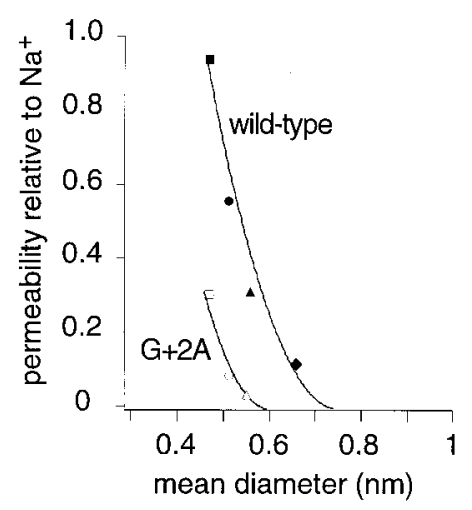

Figure 10. Determinants and dimensions of the narrow constriction. $A$, Glutamate-induced currents recorded from an outside-out patch containing GluR-D $\mathrm{D}_{\mathrm{i}} \mathrm{S}$ channels. Glutamate $(1 \mathrm{~mm})$ was applied for $50 \mathrm{msec}$ (thick bar) at different voltages with $100 \mathrm{~mm} \mathrm{NaCl}$ in the pipette and $100 \mathrm{~mm}$ TMA in the bath. Peak currents are plotted against the voltage (right panel, diamonds). Continuous lines in the right panel are the $I-V$ relations recorded before and after switching to TMA solution. $B$, As in $A$, but recording from a patch containing GluR- $\mathrm{D}_{i} \mathrm{~S}(\mathrm{G}+2 \mathrm{~A})$ channels. $C$, The permeability ratio of organic cations versus sodium plotted against the mean diameter of the organic cation, which are, from left to right, DMA (square), TriMA (circle), TMA (triangle), and TEA (diamond). Only organic cations with unambiguous reversal potentials are included $\left(V_{\text {rev }}>\right.$ $-100 \mathrm{mV}$ ). The data could be described with the hydrodynamic equation (lines). The intercept with the abscissa was taken as an estimate of the mean pore diameter.

sibility in AMPAR as the smaller MTSEA did in NMDAR channels, consistent with the different dimensions of the pore in the two GluR subtypes.

\section{Secondary structure of the M2 loop}

The pattern of exposed residues suggests that the ascending limb of the loop, on the $\mathrm{N}$-terminal side of the $\mathrm{Q} / \mathrm{R}$ site, forms an $\alpha$-helix, whereas the descending limb, on the $\mathrm{C}$-terminal side of the $\mathrm{Q} / \mathrm{R}$ site, forms an unstructured random coil. Inferring the secondary structure of a protein segment from the accessibility of substituted cysteines requires caution: the nonreactive positions may be exposed but not accessible to the reagents because of sterical constraints or particulars of the chemical microenviron- 
Table 3. Probing the constriction in GluR-D $\mathrm{S}$ and GluR-D $\mathrm{D}_{\mathrm{i}} \mathrm{S}(\mathrm{G}+2 \mathrm{~A})$ channels with organic cations

\begin{tabular}{|c|c|c|c|c|c|c|c|}
\hline \multirow{2}{*}{$\begin{array}{l}\text { Organic } \\
\text { cation }\end{array}$} & \multirow{2}{*}{$\begin{array}{l}\text { Junction } \\
\text { potential }\end{array}$} & \multicolumn{2}{|l|}{$V_{\text {rev }}($ mean $\pm \mathrm{SD})$} & \multicolumn{2}{|l|}{$P_{\mathrm{x}} / P_{\mathrm{Na}}$} & \multirow{2}{*}{$\begin{array}{l}\text { Diameter } \\
(\mathrm{nm})\end{array}$} & \multirow{2}{*}{$\begin{array}{l}\text { Size } \\
(\mathrm{nm} \times \mathrm{nm} \times \mathrm{nm})\end{array}$} \\
\hline & & Wild type & $\mathrm{G}+2 \mathrm{~A}$ & Wild type & $\mathrm{G}+2 \mathrm{~A}$ & & \\
\hline DMA & 1.5 & $-1.2 \pm 2.3(8)$ & $-33.3 \pm 2.9(4)$ & 0.95 & 0.27 & 0.47 & $0.38 \times 0.42 \times 0.61$ \\
\hline TriMA & 0.8 & $-16.2 \pm 2.0(6)$ & $-65.7 \pm 11.3(4)$ & 0.53 & 0.08 & 0.51 & $0.40 \times 0.54 \times 0.60$ \\
\hline TMA & 1.7 & $-33.5 \pm 3.0(11)$ & $-76.9 \pm 2.5(4)$ & 0.27 & 0.05 & 0.55 & $0.55 \times 0.55 \times 0.55$ \\
\hline TEA & 3.2 & $-58.3 \pm 4.6(6)$ & $-108.1 \pm 7.1(3)$ & 0.10 & 0.01 & 0.66 & $0.58 \times 0.70 \times 0.70$ \\
\hline TPA & 4.5 & $-103.0 \pm 9.3(4)$ & -154.5 & 0.02 & & 0.86 & $0.45 \times 1.07 \times 1.07$ \\
\hline
\end{tabular}

A

MTSES
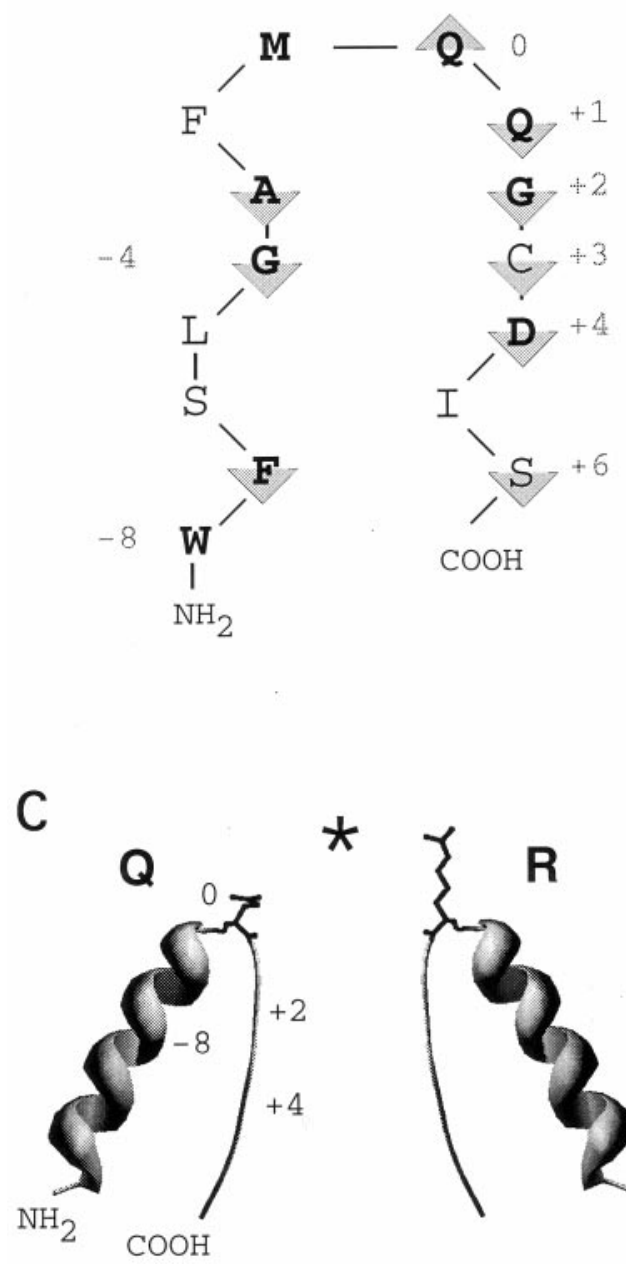

B

\section{MTSEA, MTSET}

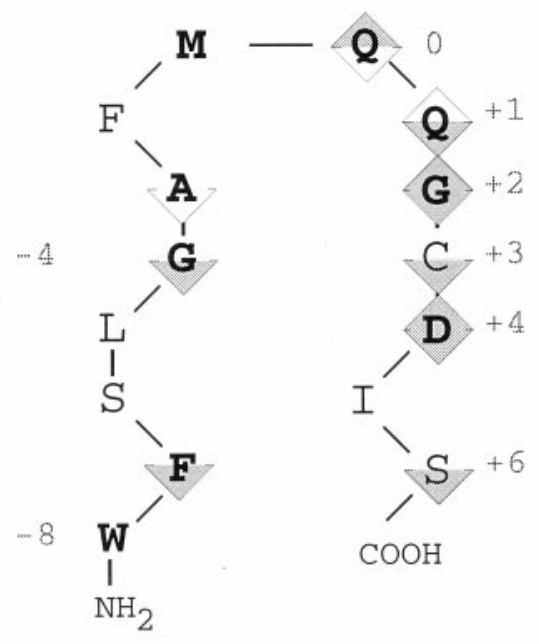

Figure 11. Models of the GluR-D $\mathrm{D}_{\mathrm{i}} \mathrm{M} 2$ segment and structural comparisons with NMDAR subunits. $A$, Two-dimensional representation of the M2 loop. Upward-pointing triangles denote positions accessible to extracellularly applied MTSES; downward-pointing triangles denote positions accessible to cytoplasmically applied MTSES. B, As in A, but showing accessibility patterns for MTSET (shaded triangles) and MTSEA (shaded and open triangles). $C$, Structural model of the M2 segment, location of the narrow constriction, and positioning of the residues occupying the $\mathrm{Q} / \mathrm{R}$ site (black side chains). The asterisk denotes the projection point of the helix dipole. Numbers denote positions relative to the $\mathrm{Q} / \mathrm{R}$ site. D, Comparison of the patterns of exposed residues in GluR-D $\mathrm{D}_{\mathrm{i}}, \mathrm{NR} 1$, and NR2 subunits. Exposed residues are printed boldface. A consensus pattern of exposed residues for GluRs is shown below the alignment; filled diamonds denote channel-lining positions.

ment. Another possibility is that a reaction occurred without affecting the function of the protein. In addition, the patterns shown in Figures 9 and 11 may arise from two different activation states of the channel each having a distinct pattern of exposed residues, because the reagents were applied in the presence of agonist when channels exist in both open and closed conforma- tions. Although in this study only kainate was used to activate GluR-D $D_{i}$ channels, other agonists may produce distinct accessibility patterns that may arise from different degrees of closure of the agonist-binding domain (Armstrong and Gouaux, 2000), consistent with the observation that different subconductance levels are elicited by the agonists glutamate and kainate (Swanson et al., 1997). 


\section{Location of the narrow constriction and positioning of the Q/R site}

The results demonstrate that the narrow constriction in AMPAR channels is located at and adjacent to the +2 position. The main evidence derives from the finding that adding a methyl group at the +2 position led to a decrease in pore diameter by $\sim 0.17 \mathrm{~nm}$. Methyl groups projecting into the lumen perpendicularly to the channel axis predict a decrease of the pore diameter by $\sim 0.3 \mathrm{~nm}$. The difference between the predicted and the measured diameters suggests that the methyl side chains are positioned at an angle, or that they produce a local conformational change within the random coil that could result in the repositioning of main chain carbonyl groups facing the pore. Such a location of the narrow constriction is consistent with the accessibility patterns of the MTS reagents, although they suggest that a narrow region may extend from positions +1 to +3 .

Position $0(\mathrm{Q} / \mathrm{R}$ site) is located on the external side of the narrow constriction, providing the possibility that the side chain of the residue at the $\mathrm{Q} / \mathrm{R}$ site projects into the extracellular vestibule of the channel (Fig. 11C). Such an arrangement is consistent with the finding that the diameter of the pore is independent of the identity of the residue present at the $Q / R$ site (Burnashev et al., 1996). In our model, the Q/R site overlooks the entrance to the narrow constriction (Fig. 11C) and is therefore ideally positioned to control passage of ions into the constriction. Position +4 may line the internal side of the narrow constriction, consistent with the observation that the MTS reagents reacted most rapidly with a cysteine residue at position +4 when applied from the cytoplasmic side. Hence, the narrow constriction seems to be bracketed by two functionally important positions: the Q/R site on its external side and an aspartate residue at position +4 on its internal side.

\section{Charge selectivity and anion permeability}

The negatively charged MTSES reacted with positions at the narrow constriction, suggesting that anions can enter deep into the cation-selective AMPAR channel. This is consistent with the finding that AMPAR channels with an arginine residue at position 0 exhibit a weak permeability for $\mathrm{Cl}^{-}$(Burnashev et al., 1996). MTSES reacted with a cysteine at position 0 only from the extracellular side and with a cysteine at position +1 only from the cytoplasmic side, suggesting that charge selectivity occurs slightly external to the narrow constriction. One intriguing possibility could be that in analogy to potassium channels (Doyle et al., 1998), the negative dipole (Roux and MacKinnon, 1999) of the pore helix (here formed by residues on the $\mathrm{N}$-terminal side of position 0) projects into the space just external to the narrow constriction, where the side chains of the residues at position 0 might be located (Fig. 11C). With a glutamine residue at position 0 , the negative electrostatic potential would attract cations to the selectivity filter and repel anions. In contrast, arginine side chains at position 0 would neutralize the negative electrostatic potential and even create a net positive potential, thereby facilitating anion permeation. The negative electrostatic potential of the helix dipole could be thought of as stabilizing the argininyl group within the space slightly external to the narrow constriction (Fig. 11C), preventing divalent and attenuating monovalent ion flux. Such a configuration of the pore loop raises the possibility that the selectivity filter of AMPAR channels is formed by two additive mechanisms: an electrostatic mechanism operating external to a narrow constriction to confer charge selectivity and a size- selective mechanism operating at the narrow constriction to select among cations.

\section{Comparison with NMDAR channels}

The patterns of exposed residues in the M2 segment of the

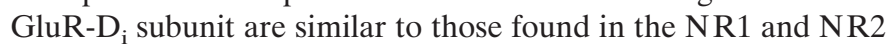
subunits (Fig. 11D). In both AMPAR and NMDAR subunits, the $\mathrm{N}$-terminal region of position 0 is consistent with an $\alpha$-helical pattern, whereas the $\mathrm{C}$-terminal region suggests a random coil. A consensus pattern of exposed residues for the M2 segment of the glutamate receptor family is shown in Figure $11 D$ (diamonds). The subunits show a similar positioning of the residue at the 0 position, two-sided accessibility at positions +1 to +4 , and restricted accessibility at position -3 . Nevertheless, the GluR-D pattern is more similar to the NR2 pattern than to the NR1 pattern, consistent with a specialized role of the NR1 subunit in creating an asymmetric pore (Kuner et al., 1996). In conclusion, AMPARs possess pore loops with a structural design similar to that of NMDAR channels but different sets of amino acid residues creating different functional profiles in the two receptor subtypes.

\section{REFERENCES}

Akabas MH, Stauffer DA, Xu M, Karlin A (1992) Acetylcholine receptor channel structure probed in cysteine-substitution mutants. Science 258:307-310.

Akabas MH, Kaufmann C, Archdeacon P, Karlin A (1994) Identification of acetylcholine receptor channel-lining residues in the entire M2 segment of the alpha subunit. Neuron 13:919-927.

Armstrong N, Gouaux E (2000) Mechanisms for activation and antagonism of an AMPA-sensitive glutamate receptor: crystal structures of the GluR2 ligand binding core. Neuron 28:165-181.

Ausubel FM, Brent R, Kingston RE, Moore DD, Seidman JG, Smith JA, Struhl K (1995) Mutagenesis of cloned DNA. In: Current protocols in molecular biology, Ch 8 (Janssen K, ed), pp 233-250. New York: Wiley.

Beck C, Wollmuth LP, Seeburg PH, Sakmann B, Kuner T (1999) NMDAR channel segments forming the extracellular vestibule inferred from the accessibility of substituted cysteines. Neuron 22:559-570.

Blaschke M, Keller BU, Rivosecchi R, Hollmann M, Heinemann S, Konnerth A (1993) A single amino acid determines the subunitspecific spider toxin block of alpha-amino-3-hydroxy-5-methylisoxazole-4-propionate/kainate receptor channels. Proc Natl Acad Sci USA 90:6528-6532.

Bowie D, Mayer ML (1995) Inward rectification of both AMPA and kainate subtype glutamate receptors generated by polyamine-mediated ion channel block. Neuron 15:453-462.

Burnashev N, Monyer H, Seeburg PH, Sakmann B (1992) Divalent ion permeability of AMPA receptor channels is dominated by the edited form of a single subunit. Neuron 8:189-198.

Burnashev N, Villarroel A, Sakmann B (1996) Dimensions and ion selectivity of recombinant AMPA and kainate receptor channels and their dependence on Q/R site residues. J Physiol (Lond) 496:165-173.

Colquhoun D, Jonas P, Sakmann B (1992) Action of brief pulses of glutamate on AMPA/kainate receptors in patches from different neurones of rat hippocampal slices. J Physiol (Lond) 458:261-287.

Dingledine R, Hume RI, Heinemann SF (1992) Structural determinants of barium permeation and rectification in non-NMDA glutamate receptor channels. J Neurosci 12:4080-4087.

Dingledine R, Borges K, Bowie D, Traynelis SF (1999) The glutamate receptor ion channels. Pharmacol Rev 51:7-61.

Donevan SD, Rogawski MA (1995) Intracellular polyamines mediate inward rectification of $\mathrm{Ca}^{2+}$-permeable alpha-amino-3-hydroxy-5methyl-4-isoxazolepropionic acid receptors. Proc Natl Acad Sci USA 92:9298-9302.

Doyle DA, Cabral JM, Pfuetzner RA, Kuo A, Gulbis JM, Cohen SL, Chait BT, MacKinnon R (1998) The structure of the potassium channel: molecular basis of $\mathrm{K}^{+}$conduction and selectivity. Science 280:69-77.

Dwyer TM, Adams DJ, Hille B (1980) The permeability of the endplate channel to organic cations in frog muscle. J Gen Physiol 75:469-492.

Geiger JR, Melcher T, Koh DS, Sakmann B, Seeburg PH, Jonas P, Monyer H (1995) Relative abundance of subunit mRNAs determines gating and $\mathrm{Ca}^{2+}$ permeability of AMPA receptors in principal neurons and interneurons in rat CNS. Neuron 15:193-204.

Hamill OP, Marty A, Neher E, Sakmann B, Sigworth FJ (1981) Im- 
proved patch-clamp technique for high resolution current recording from cells and cell-free membrane patches. Pflügers Arch 391:85-100.

Holmgren M, Liu Y, Xu Y, Yellen G (1996) On the use of thiolmodifying agents to determine channel topology. Neuropharmacology 35:797-804.

Hume RI, Dingledine R, Heinemann SF (1991) Identification of a site in glutamate receptor subunits that controls calcium permeability. Science 253:1028-1031.

Isa T, Iino M, Itazawa S, Ozawa S (1995) Spermine mediates inward rectification of $\mathrm{Ca}^{2+}$-permeable AMPA receptor channels. NeuroReport 6:2045-2048.

Kamboj SK, Swanson GT, Cull CS (1995) Intracellular spermine confers rectification on rat calcium-permeable AMPA and kainate receptors. J Physiol (Lond) 297-303.

Koh DS, Burnashev N, Jonas P (1995) Block of native $\mathrm{Ca}^{2+}$ permeable AMPA receptors in rat brain by intracellular polyamines generates double rectification. J Physiol (Lond) 486:305-312.

Kuner T, Schoepfer R (1996) Multiple structural elements determine subunit-specificity of $\mathrm{Mg}^{2+}$ block in NMDA receptor channels. J Neurosci 16:3549-3558.

Kuner T, Wollmuth LP, Karlin A, Seeburg PH, Sakmann B (1996) Structure of the NMDA receptor channel M2 segment inferred from the accessibility of substituted cysteines. Neuron 17:343-352.

Kuner T, Beck C, Seeburg PH, Sakmann B (1997) Pore-lining residues of the AMPA receptor channel M2 segment. Soc Neurosci Abstr 23:925.

Liu SQ, Cull-Candy SG (2000) Synaptic activity at calcium-permeable AMPA receptors induces a switch in receptor subtype. Nature 405:454-458.

MacKinnon R, Yellen G (1990) Mutations affecting TEA blockade and ion permeation in voltage-activated $\mathrm{K}^{+}$channels. Science 250:276-279.

Mosbacher J, Schoepfer R, Monyer H, Burnashev N, Seeburg PH, Ruppersberg JP (1994) A molecular determinant for submillisecond desensitization in glutamate receptors. Science 266:1059-1062.

Roux B, MacKinnon R (1999) The cavity and pore helices in the KcsA $\mathrm{K}+$ channel: electrostatic stabilization of monovalent cations. Science 285:100-102.

Swanson GT, Feldmeyer D, Kaneda M, Cull-Candy SG (1996) Effect of RNA editing and subunit co-assembly single-channel properties of recombinant kainate receptors. J Physiol (Lond) 129-142.

Swanson GT, Kamboj SK, Cull-Candy SG (1997) Single-channel properties of recombinant AMPA receptors depend on RNA editing, splice variation, and subunit composition. J Neurosci 17:58-69.

Verdoorn TA, Burnashev N, Monyer H, Seeburg PH, Sakmann B (1991) Structural determinants of ion flow through recombinant glutamate receptor channels. Science 252:1715-1718.

Villarroel A, Burnashev N, Sakmann B (1995) Dimensions of the narrow portion of a recombinant NMDA receptor channel. Biophys $\mathbf{J}$ 68:866-875. 